Response to Commentaries on A Life-Saving Therapy:

The Theory-Building Case of "Cora"

\title{
Conceptualizing the Complexity of Change in Psychotherapy: The Case of "Cora”
}

\section{MARGRETHE SEEGER HALVORSEN, ${ }^{\mathrm{a}, \mathrm{b}}$ JOHN MCLEOD, ${ }^{\mathrm{a}}$ KIRSTEN BENUM, ${ }^{\mathrm{a}}$ HANNE HAAVIND ${ }^{\mathrm{a}}$}

a Department of Psychology, University of Oslo, Norway

${ }^{\mathrm{b}}$ Correspondence regarding this article should be sent to Margrethe Seeger Halvorsen, Department of Psychology, University of Oslo, Pb. 1094 Blindern, 0317 Oslo, Norway

Email: m.s.halvorsen@psykologi.uio.no

\begin{abstract}
In this article we respond to the commentaries by Altman and Miller (2016), Bohart (2016), and Timulak and Keogh (2016) on our case of “Cora," which involved over 121 psychotherapy sessions with a client with a history of severe relational trauma that had led to difficulty in forming close relationships, as well as a series of suicide attempts (Halvorsen, Benum, Haavind, \& McLeod, 2016). The commentaries differ in their view of the value of Cora's case, interpretations of the material, and methodological preferences. Together the commentaries illustrate the complexity of change in psychotherapy, how we need multifaceted approaches to understand change, and the challenges in writing up a case in this context. We call for further dialogue within the psychotherapy research community around the nature and implications of methodological choices arising from the conduct of case study research in this field.
\end{abstract}

Key words: case study; clinical case study; mixed method; complexity of change; psychotherapy; courage; theory-building case; symbolic objects in psychotherapy

\section{INTRODUCTION}

We wish to thank the commentators (Altman \& Miller, 2016; Bohart, 2016; and Timulak \& Keogh, 2016) for their thoughtful and stimulating reviews of our case of Cora (Halvorsen, Benum, Haavind, \& McLeod, 2016). Although the three commentaries differ somewhat in respect of the specific aspects of the case on which they have chosen to focus, we believe that each of them draws attention, in their own way, to fundamental issues associated with the challenges associated with conceptualizing complex change processes in psychotherapy. As a result, rather than address the contribution of each commentary in turn, we wish to use our rejoinder to explore common themes and questions that emerge from their generous and constructive engagement with our work. 
In the following paragraphs, we invite readers to reflect on what is happening when a psychotherapy case study is produced/written, consumed/read, and the knowledge from the case is then used or applied. We believe that the notion of "conceptualizing” reflects a crucial and central aspect of all stages of this process. In the discussion that follows, we treat both methodological and clinical aspects of the case of Cora as inter-connected aspects of a more general professional task of conceptualizing or "making sense”. Rather than adopting a rigid distinction between the methodological issues and the substantive/clinical issues raised by Altman and Miller (2016), Bohart (2016), and Timulak and Keogh (2016), we illustrate some of the ways in which clinical-theoretical assumptions and choices shaped our approach to analyzing the data in this case, and epistemological choices shape our approach to the clinical meaning of the case.

\section{WHAT KIND OF A CASE IS IT?}

If psychotherapy is understood as a complex intervention (Craig et al., 2008), it follows that it is necessary to implement a range of methodologies in order to arrive at reliable knowledge of how it works and how its effectiveness might be enhanced. This position is widely accepted within the psychotherapy community, and in relation to research it has led to an acknowledgement of the value of methodological pluralism (Slife \& Gantt, 1999). This requires an understanding of the strengths and limitations of different methodological approaches, such as randomized trials, qualitative interview studies, and case studies. In terms of the array of methodologies used in contemporary psychotherapy research, the distinctive strengths of case study inquiry include a capacity to investigate complexity, an ability to take account of contextual factors, sensitivity to client and therapy agency, analysis of how processes unfold over time, and memorability of findings (McLeod, 2010).

Within the field of case study methodology itself, it is possible to identify four different types of case investigation: outcome-oriented, narrative, pragmatic, and theory-building (McLeod, 2011, 2013). Each of these case-based methodologies has its own strengths and limitations, and is associated with a particular style of case analysis. However, there is inevitable some ambiguity associated with this typology, because it is hard to write a satisfying case report without, to some extent, making use of all four perspectives. So, for example, although our main aim was to contribute to theoretical understanding or theory-building, in order to allow readers to make sense of the case as a whole, we also needed to write about the story or narrative of the case, the professional framework within which therapy was conducted (as in a pragmatic case study), and the question of whether it was a good or poor outcome case.

We suggest that some of the possible frustration experienced by commentators on the case, and by general readers, arises from the fact that they had questions about the case that were not adequately addressed in the particular case report that we chose to write. For example, Altman and Miller (2016) were disappointed that "the case itself feels strangely ephemeral” (p. 217) because the personal experience of the therapist and client was not sufficiently highlighted. Bohart (2016) and Timulak and Keogh (2016) suggest that important dimensions of the case might have been better understood through the application of a jury method, or the use of Hermeneutic Single Case Efficacy Design (Elliott, 2002). We agree with these comments, and we hope that we made it clear in our original paper that other ways of working with this case 
material would certainly yield further insights. At the same time, it is important to be realistic about what can be achieved in a single study. Approaching the case of Cora through the lens of a narrative analysis or outcome-focused analysis, would involve a fundamental re-analysis of the data. These would certainly be interesting and valuable projects, but to do either of them properly would probably require the use of an additional team of researchers who were able to concentrate solely on those aspects of the data that were most relevant to their goals.

Altman and Miller (2016) point out that our study "raises more theoretical questions than it answers in terms of our ability to enumerate the conceptual elements necessary to convey valuable clinical truths” (p. 215). They seem to favor a different type of case study, written by the therapist himself, rather than us as external researchers. This is an interesting methodological point of view, which is contrary to what we usually have heard from reviewers and other audiences, as well as what we read in the literature on case study research. Altman and Miller (2016) seem to claim that the majority of case studies are written by the therapist himself or herself. We firmly disagree with this. The classical case studies were usually written by the therapist himself (e.g., Freud's case studies), but in the latest decades the trend seems to have moved toward researchers looking into other therapists' work. No doubt that there is much to learn from the therapist himself in our case, but that would be another study. Unfortunately, due to the therapist's current health condition he is not able to respond to this commentary, but what we do know is that he was very proud of his work with Cora, and that he wanted us to write up a case on this therapeutic process, even though he did not want to be part of the writing process himself.

In the light of these comments about the merits of adopting different styles of case inquiry, the further development of case study research in psychotherapy might be facilitated by considering the methodological learning that could arise from multiple analyses of the same case. Although this approach has historically been part of the psychoanalytic tradition, for example in relation to the cases published by Freud, and more recently in the form of the case of Lisa (Angus, Goldman \& Mergenthaler, 2008), there have been few attempts to evaluate the differential yield of alternative methods of analyzing case data (Greenberg, 2008).

One of the key ways in which different case study methodologies can be contrasted, is suggested by Altman and Miller (2016) in their discussion of the authorial "voice" in which the case is written. As mentioned, most psychotherapy research papers are written using a detached, expert-scientific voice (Gergen, 1997), and this is the main voice that we adopt in our report on the case of Cora. It is clear that in case study inquiry it can be informative to communicate using other voices, such as the voice of the therapist and the client. We believe that there are complex issues around the question of the voice through which case studies are written. For example, there is a difference between the voice of the client expressed in a piece of writing that is entirely within the control of the client, and the voice of the client expressed in segments of interview narrative that are selected and edited by a researcher or their therapist. Similarly, there is a difference between the clinical-professional voice that is used in most case studies written by therapists, and the more personal voice that the same individual might use when describing the same case in a different context. We do not accept the kind of privileging of the therapist voice that appear to be suggested by Altman and Miller (2016), because our own response to many case studies we read is to ask the question: "What did the client make of all this?" However, we 
wholly agree that authorial voice is an important topic for further discussion, innovation and evaluation within the field of psychotherapy case study writing.

The significance of authorial voice becomes clearer through reflection on the links between research and practice that are opened up by case study research. Most therapy research studies with group designs conclude with a set of findings that function as candidate practice guidelines. A reader of such a study is faced with the task of, first, deciding on whether the methodology of the study is valid, and then reflecting on the practice implications of the headline findings. By contrast, the meaning of a case study lies in the case report as a whole, because any conclusions or findings only make sense in that context. As a result, to learn from a case study, it is necessary to take time to imaginatively enter the world of the case. It is of interest that all three sets of commentators on the Cora case mentioned their own subjective response to the case, and their reading was informed by recollection of similar cases and clinical issues that they had encountered in their personal experience. It seems plausible that case studies that are written in ways that convey multi-faceted therapist and client voices may be more generative for clinicianreaders, in evoking such personal material. On the other hand, such readers may fail to be convinced by case material that they regard as failing to represent the case in an even-handed, comprehensive manner. There are undoubtedly strategies for achieving balance between multivoicedness and methodological rigor that remains to be discovered.

In relation to the question of the kind of case study represented in our analysis of how the therapist and the client engaged themselves in interactions in sessions over time, we were appropriately taken to task by Timulak and Keogh (2016) around our claim to have offered a theory-building case. These commentators correctly point out that the model of theory-building case research outlined by Stiles (2007) calls on researchers to apply the case to the theory, a process that requires carefully articulating the theory in advance of the case analysis, and making sure that the case analysis addressed all possible points of contact between the theory and the case data. Timulak and Keogh (2016) usefully draw attention to the ways in which such an approach can contribute to the development of practice-relevant theoretical knowledge.

While acknowledging the value of the model of theory-building research developed by Stiles (2007), we would point out that other approaches to the theory-building do exist (see, for example, Fishman \& Westerman, 2011; Swedberg, 2014). Within the field of qualitative research, it is possible to view themes or categories as potential theoretical constructs, or as means of developing an enriched understanding of established constructs. The qualitative methodology that we used to frame our process of inquiry (Interpretative Phenomenological Analysis; IPA, Smith, Flowers, \& Larkin, 2009) explicitly encourages such a step.

Looking back, it is always possible to identify aspects of a research report that could have been explained in more detail. Ideally, we would have liked to have written a lot more about our theory-building hopes and intentions in the Cora case, but were inhibited by the risk of testing the patience of our readers. What we were trying to say, right at the start of the Cora paper, was that we hoped to use the case to advance our theoretical understanding of the nature of therapist expertise, by looking at what happened when a clinician who could be credibly defined as expert on the basis of reputation and experience, was faced with a client who was hard to treat. This seemed to us to comprise a theoretically fruitful line of inquiry, on the grounds that a competent 
therapist might achieve good results with clients who were ready to change, and could draw on a range of resources and success experiences. By contrast, a client with longstanding problems and a wish to kill herself would present a stern challenge for any clinician. In addition, we wondered whether in-depth case analysis might open up ways of understanding therapist expertise that might be harder to identify through large-n studies using measures.

At the end of the day, we are cautiously confident that our analysis of the Cora case provides tentative evidence for three characteristics of therapy expertise (persistence, courage, and symbolic confirmation) that have not been found in large-scale studies. We entirely agree that the nature, origins, and representativeness of such qualities needs to be explored and tested in further research, using a range of methodologies.

A final point about theory-building research is to consider the implications of restricting theory-building to studies that are designed to elaborate on existing, pre-defined theories. We do not believe that Stiles (2007) calls for such a thing. Nevertheless, we suggest that any such policy runs the risk of inhibiting the development of new ideas. We believe that it is essential those conducting case study research to be open to whatever a case may say to them:

researchers who have conducted intensive, in-depth case studies typically report that their preconceived views, assumptions, concepts, and hypotheses were wrong and that the case material has compelled them to revise their hypotheses on essential points (Flyvbjerg, 2006: 235).

For example, what this meant for us was that we were surprised by the emergence of the theme of courage. This was an idea that we would not have included in a model of therapist expertise, in advance of undertaking the case analysis. At various points in the process of analyzing the data were uncomfortable about this concept, which seemed to imply a moral dimension that was hard to reconcile with the prevailing wisdom about what is important in psychotherapy.

\section{WHAT IS THE BEST WAY TO ANALYZE AND PRESENT CASE STUDY DATA?}

The commentary by Timulak and Keogh (2016) includes a thoughtful and insightful critique of our use of IPA as a framework to guide our data analysis. Timulak and Keogh question why we used IPA in a process study like this. Is it the most proper method? It is true that IPA most often is used on interview data, but as we mention IPA has also been applied, successfully in our view, in a number of case study investigations (Bramley \& Eatough, 2005; Eatough \& Smith, 2006a,b; Quinn, Schofield, \& Middleton, 2012). We also agree that IPA overlaps significantly with other descriptive interpretative methods. We do not see any contradiction here. In our view the use of IPA on process material is an extension of the traditional use of IPA, and in this case we used the method as a guide into the material in combination with the existing material and our understanding of it.

Timulak and Keogh (2016) argue that our adoption of a thematic approach such as IPA had the effect of obscuring potentially significant aspects of the case, such as progress across stages of therapy, and that the structure of IPA may have resulted in downplaying the 
significance of other processes or themes that might have been analyzed, as well as failing to clarify the relative weight given to different themes. We regard these observations as constructive and helpful, in relation to the design of future case study analyses that we intend to conduct. For example, we did analyze the data in terms of stages of therapy, but this perspective was not emphasized in the final published version of the findings. However, we wish to add that we also believe that there are positive and negative aspects of any approach that currently exists, in respect of analyzing mixed qualitative and quantitative data in cases of long-term therapy. We suggest that it would be useful for the research community to engage in further dialogue and experimentation around ways of analyzing and presenting case study reports. Within the domain of Hermeneutic Single Case Efficacy Design (HSCED) research, there have been two review papers that have reflected on the advantages and disadvantages of different procedures that have been deployed in studies using that approach (Benelli, De Carlo, Biffi, \& McLeod, 2015; Wall, Kwee, Hu \& McDonald, 2016). It would be helpful to conduct a similar exercise within the domain of theory-building case study research, as a means of taking stock of where we have reached, and the methodological options that are available.

Earlier versions of the Cora case included much more comprehensive explanation and rationale around the steps that we took in order to analyze the data. It took a long time to analyze the case, and the process was much messier than the summary included in the article published in the present issue of this PCSP journal. We would argue that it is possible to identify something of a crisis or impasse in qualitative research at the present time. On the one hand, journal editors and reviewers - and some readers - are reassured by the idea that a study has adhered to a clearly-defined set of analytic procedures, which they can learn about by accessing the appropriate sources. On the other hand, experienced qualitative researchers know that nothing is ever that simple, and want to learn about what the research team actually did with the data. In our account of the case of Cora, we decided that it was more meaningful for us to share what we had learned (or what we thought we had learned) from the case in relation to the question of how change can occur in an unlikely circumstances. We believed that too much methodological detail might detract from this purpose.

An aspect of the methodology that is perhaps not sufficiently highlighted in Halvorsen et al. (2016), and which we could like to emphasize now, is that the case analysis started at the end and worked backwards. In other words, we began by using IPA to analyze the type of data to which it has been applied in innumerable studies (i.e., end-of-therapy interview transcripts) and then stretched it by looking at other data (mainly session transcripts and open-ended written reports) to build an interpretative account of what it was that appeared to lead to these outcomes, or how these end-point meanings were grounded in earlier dialogue. This strategy privileges the perspectives of the client and therapist as interpreters of their own experience-a standpoint that is central to IPA methodology.

Another aspect of our methodology that is not emphasized in Halvorsen et al. (2016) is the extent to which the research process required us to consider, and discuss as a group, fundamental epistemological questions about our capacity to understand the material at all, and the grounds on which our coding and interpretation might be warranted. Another way of framing this thread of the work is to acknowledge that it was informed as much by philosophical considerations as by methodological ones. In this respect, the choice of IPA was appropriate and 
helpful for us, because of the careful way in which Smith, Flowers and Larkin (2009) elucidate the philosophical origins of their method.

A final aspect of the methodology that can be mentioned here is the extent to which the process of analysis involved active reflexivity on the part of members of the research group. If our meetings had been recorded and coded, it would have been apparent that there were many occasions where episodes in the Cora case evoked memories of our experience as clinicians or clients. Typically, these experiences would be briefly described and then interrogated in terms of what they might tell us about ambiguous aspects of the data. Another feature of the reflexive or personal dimension of the process of analysis was reflected in sensitivity toward other members of the group, for example around questioning their interpretation of segments of the data or finding theme titles that adequately reflected nuances of understanding that were significant to all of us.

Timulak and Keogh (2016) pinpoint the challenges involved, in writing up a case, of resolving the tension between subjective and objective responses to the material. It is definitely true that we probably were affected by the poignancy of this case, and that we could be more explicit about how this informed us. It was encouraging to learn that Timulak and Keogh (2016) regard subjectivity as a strength, rather than a shortfall, in the context of a case study such as this. We agree with them that researchers are professionally socialized to appear rigorously objective, and as a result become wary of making constructive use of subjective responses to case material.

\section{MAKING SENSE OF DISCREPANCIES BETWEEN QUALITATIVE AND QUANTITATIVE FINDINGS}

All of the commentaries reflected on the intriguing finding, in the Cora case, that qualitative and quantitative sources of evidence provided strikingly different estimates of change. In particular, Timulak and Keogh (2016) offer a valuable review on how these two variants of self-report may be influenced by different factors, as well as the possibility that a client's experiences of change in other domains than what is measured by the questionnaires (e.g., suicidal ideation, self-harm, or risk) can be taken as evidence of change.

In the original case report (Halvorsen et al., 2016), we offer our own interpretation of this phenomenon. In retrospect, we can see that it could have been valuable to have devoted more time and effort to resolving this discrepancy, for example by searching transcripts for further corroborating evidence, as suggested by Bohart (2016), or by implementing some form of HSCED or other jury-based analysis, as suggested by both Bohart (2016) and Timulak and Keogh (2016).

In terms of the process of carrying out the case analysis, at the outset most of our resources were devoted to the task of analyzing the qualitative data, and we adopted the view that the quantitative data from standardized measures merely provided contextual information, for example by allowing us to position the case within the wider sample of our overall study, and within the even broader context of the general literature that had made use of the same measures. It was only toward the end of our work on the case, when we began to assemble the findings into an article to be submitted for publication, that the contrast between the statistical and narrative 
aspects of the case jumped into focus.

There are three main observations that we would like to make about this issue. First, we predict that, as more mixed-method psychotherapy case-based research is carried out, this issue is destined to become more salient. Second, we do not believe that is helpful to regard discrepancies between qualitative and quantitative evidence as a matter of one type of evidence being valid and the other as wrong (in our experience, this argument can be forcefully advanced by adherents from either camp). Instead, each source of evidence needs to be approached from an appreciative but also critical stance. Third, the existence of differences in results based on different methods of measurement needs to be viewed as an awkward gift, rather than as problematic. The interpretation of complex case material always involves a process of triangulation, in which one source of evidence is used to corroborate conclusions drawn from other sources. Situations in which corroboration does not occur function as drivers to look more closely at the data, in a search for other relevant observations, or to find new ways to make sense of what has been found.

Most readers of therapy case studies are primarily interested in the actual substantive findings that are on offer. However, it is possible that, over time, measurement-method discrepancies in mixed methods case analyses may have a major impact on the profession. At the moment, we just do not know how often such divergencies occur. It seems possible that, in the past, case study authors may have been reluctant to report such findings, for fear of being accused of error. We do not know, either, whether measurement-method discrepancies are randomly distributed, or follow a pattern. If it turns out that discrepancies occur in a significant proportion of carefully analyzed, mixed-methods cases, or that they are associated with a particular type of case, then this would cast doubt on the credibility evidence that has been used to support the development of clinical practice guidelines and other aspects of service delivery and policy.

\section{CONCEPTUALIZING THE PROCESS OF CHANGE}

All of the commentaries offered further reflections on the meaning and significance of our main findings, and their implications for practice. We were delighted to read these reflections, because we regard them as evidence that we provided sufficient rich detail to enable readers to arrive at their own conclusions about the case. In addition, we believe that all theories of therapy can be regarded as part of a more general therapeutic discourse that has been developed within contemporary culture. Rather than adopting an "either/or" position in which insights from one theoretical approach require repudiation of ideas from an alternative approach, we prefer a "both/and" stance that seeks to create meaning bridges and dialogue across different professional communities.

We were a bit puzzled by Bohart's (2016) assumption that we (the authors) are embedded in a psychodynamic tradition. As noted in the manuscript, none of us has a primary theoretical affiliation in psychodynamic theory, even though some of us have been inspired and influenced by psychodynamic thinking, in addition to other perspectives. So we wonder if Bohart (2016) had read our case with a kind of psychodynamic expectation. We clearly find his reflections on childhood trauma thought provoking, but we do not recognize ourselves in his interpretation of 
how we understand childhood trauma and possible consequences later in life. We fully agree with him that living in an abusive environment for year after year may cause damage, regardless if it is experienced before age of three or after. Thus, we want to clarify our theoretical perspective regarding early adverse childhood experiences. In this respect we rely on the extensive body of research on how early relational trauma is associated with later problems with affect regulation, regulation of self and other, increased vulnerability for stress and strains (Liotti, 2006; Lyons-Ruth, Dutra, Schuder, \& Bianchi, 2006; Shore, 2003), as well as challenges in building trust and a therapeutic bond (Ogden \& Fisher, 2015; Porges, 2001).

It was inspiring to read that Bohart (2016) found our concept of persistence important. We believe that this may be a fundamental aspect of change in many therapies. Persistence is, in our view, something more than commitment, as Altman and Miller (2016) suggest as an alternative. It is an enduring interactive repetition of something meaningful that seems to have a redefining and developmental function.

We agree with Bohart (2016) that clients are more courageous than we give them credit for, and we do agree that Cora was courageous before she came into therapy. Bohart's interpretations of Cora's courage are very compelling. In his commentary he writes that the therapist (and authors) view Cora's defenses as survival strategies primarily employed to avoid painful experiences and feelings per se. This is not exactly what we mean. We agree with Bohart's notion that Cora has lived her life and survived with terribly disruptive and painful emotions. So maybe it is more in the conceptualization of affect tolerance that we disagree on, and how to understand the distinction between affect activation and affect integration (Solbakken, Sandvik Hansen, \& Monsen, 2011). Cora has clearly experienced painful emotions, over and over again, but it seems that she has been overwhelmed by them and often reacted to them (e.g., by self-harm), not being able to regulate them. Through therapy she gradually succeeded in managing and regulating her painful feelings in a better way. For us, this is an example of increased affect tolerance.

We appreciated Timulak and Keogh’s (2016) thoughtful and encouraging comments on the clinical aspects of our case. We appreciate their commentaries on the case as having a valuable role in enriching our thinking about psychotherapy. We agree that the case is not only a lifesaving therapy, but also a life changing therapy. They seem to have grasped our intention with the case, and we are pleased to read that they value the serious commitment of both parties in this case. We appreciate their insightful comments on the therapist's flexibility regarding certain boundaries and that going beyond standard protocols may in certain instances be helpful for the client in building trust. This is an unconventional and difficult topic, not often addressed either in clinics or in research, but we believe that the field needs to discuss how and when our protocols are therapeutic or not. In a paper by the Norwegian psychologist Tormod Stangeland (2012), entitled When the Safest Treatment is Not the Best Treatment, he proposes a distinction to clarify the intention of helping interventions in his work with adolescents with suicidal behavior: safety interventions (to insure survival) and security interventions (to increase attachment). We find this distinction useful. When do we promote security interventions, helping the client to relate and build trust, and when do we promote safety interventions, by taking over control and responsibility, and thereby possibly diminishing the client's ability to heal? These are 
demanding and delicate considerations and we believe that the case of Cora may shed light on aspects of these complex processes.

Timulak and Keogh (2016) offer alternative interpretations of how to understand the success of this case. We suggested that transference interpretations and immediacy might have been part of the process. We fully agree that the process may be understood in different terms, and as Timulak and Keogh suggest, the interaction between the two helped the client to build trust, which probably allowed her to take further emotional risks. Maybe we have not been precise enough in our description of how we understand the case, or that the use of the concept of "interpretation" narrows the scope. Our understanding of the case is in line with Timulak and Keogh when they claim that what might be the primary mechanism here is the client's experience of being cared for and having her needs met, not merely a deepened understanding through the therapist's interpretations.

The existence of multiple plausible understandings of these various aspects of the case of Cora is consistent with an appreciation of the complexity of process and outcome in psychotherapy. For example, at any point in therapy, a clinician is likely to be engaging in a wide range of potentially helpful processes: empathic attunement, reflection on the meaning of what the client is talking about, self-monitoring his or her own internal responses to the client, responsive non-verbal gestures and body movements, and much else (McLeod, 2013). As a result, it is extremely difficult to identify, in relation to observed client change, which of these activities had a causal effect. The field of complexity science offers a range of approaches to making sense of complexity in clinical practice (Plsek \& Greenhalgh, 2001; Wilson \& Holt, 2001). A set of thoughtful examples of how the question of outcome in specific cases of psychotherapy can be understood in multiple ways can be found in Ben-Shar and Shalit (2016).

One of the distinctive characteristics of case study research lies in the extent to which it has the potential to explore complexity, in the form of multiple co-occurring processes and changes over time. The challenge for case study researchers is to collect data that reflects complexity, while at the same time carrying out an analysis of the case in a way that is able to highlight key change processes, without overwhelming the reader with detail. This a difficult balancing act. Our main strategy for dealing with this was to view the therapist and client as the local experts on the case, and to assume that their reports could be taken to represent attempts to sift through the experienced complexity of their experience and tell us about what, from their perspective, were the processes and activities that really mattered.

Reviewers of the case have been able to "re-complexify" our account, by suggesting further ways of making sense of our themes of courage and persistence. We regard this as a positive accomplishment on our part, which bears testimony to the success of our efforts to be clear about what we agreed, after many hours of discussion, as the key themes and processes in the case, while also writing up the case in a manner that allowed other interpretations to be sustained. We suggest that a clinically satisfying case study report may be one that includes sufficient "surplus meaning" to allow readers to make up their own minds. Another criterion for a satisfying case report arises from appraisal of whether the case analysis has followed a credible and transparent process of data collection and reduction. 
In their commentary, Altman and Miller (2016) called for more information about the therapeutic style, espoused and applied theories, and professional development of the therapist in the Cora case, and offered some thoughtful reflections on the interpretative possibilities that such information might have opened up. We agree that such information would have been extremely valuable. From our own perspective, there were points in our analysis of the case where we found ourselves wishing that we had access to more information about the everyday life of Cora, to enable us to arrive at a better understanding of how such "extra-therapeutic" experiences and activities interacted with what was happening in the therapy room. The question of the adequacy of the case data, in the form of a "rich case record" (Elliott, 2002) represents a further criterion on which case study analyses can be assessed. In this context, it is of interest that even though the Cora case analysis was based on a richer data set than is available in most published psychotherapy case studies, it is still clear that it would have been useful to have had access to even more data. We suggest that an urgent task for the psychotherapy research community is to develop economical, time-sensitive and minimally intrusive means of collecting information on a wider range of conceptually relevant aspects of case-based research, such as therapist experience and characteristics, and the structure of the everyday life of the client.

\section{SYMBOLIC CONFIRMATION OF CHANGE}

Toward the end of therapy, Cora gave her therapist a series of objects, which they both understood as ways of symbolizing and representing the quality and significance of her experience of having been able to be in relationship with him. We used the concept "symbolic confirmation” to reflect our interpretation of these events. It seemed to us that symbolic confirmation was connected to the other main themes that emerged in our analysis of the case. Specifically, these exchanges confirmed the depth of persistence and courage that had occurred. In relation to this, we want to underscore that all these three themes are interactional phenomena; they represent a co-created quality of the relationship, and not primarily an attribute embedded in each person. Despite the fact that none of the commentators on the Cora case highlighted the symbolic confirmation aspect of the analysis, we would like to underscore our belief that this theme has the potential to stimulate further development in the areas of both research and practice. In Halvorsen et al. (2016), we discussed this phenomenon in terms of the meaning of gifts from clients to therapists, and now wish to add some further reflections.

Weekly, or regular, meetings with a psychotherapist can be viewed as punctuations in the everyday life of a client. During these meetings, the client is able to look back on their everyday life, reflect on its meaning, and formulate strategies for adjusting aspects of it. An important issue, from this perspective, concerns the strategies that are available to the client to retain what has been learned and experienced in the therapy room, within the context of a busy everyday life that is characterized by multiple other sources of influence. There is evidence that many clients retain an internal representation of their therapist, and may place great value on this resource as a means of support and guidance during times of stress (Geller \& Farber, 1993; Knox, Goldberg, Woodhouse, \& Hill, 1999; Mosher \& Stiles, 2009). Little attention has been devoted to the question of what the client might do to retain a memory of a significant relational connection. Råbu, Haavind, and Binder (2013) found that clients and therapists tend to generate vivid and highly memorable metaphors in the final phase of therapy, which may serve the function of facilitating future access to sustaining recollections of the therapeutic relationship. In the Cora 
case, physical objects were used as tangible markers of the relationship, with the added feature that these were given by Cora to her therapist. One of the objects - the box-was given on the understanding that it could be reclaimed at some time in the future. With another of the objectsthe carabiner-we assume that Cora would have in her own possession an identical item that would serve as a reminder. The significance of these symbolic objects is reinforced by the fact that they are versions of objects (i.e., letters, boxes and rings) that widely used within their shared cultural context to designate close abiding relationships between individuals. For example, a ring may be used to designate marriage, a commitment to marry, or the loss of a loved one.

In our view, it makes a lot of sense that someone like Cora, who has struggled through her life to be able to trust someone, would not want to walk away from this therapist, with only memories. This makes us curious about how often clients engage in such actions, and whether it is possible or helpful for their therapists to encourage such processes. This topic appears to be us to be particularly relevant in respect of therapy in which the relationship between client and therapist has been used as a "relational training ground" (Hill et al., 2008), and even more so when that learning has concerned the development of a capacity for intimacy and trust.

\section{CONCLUSION}

We believe that the commentaries by Altman and Miller (2016), Bohart (2016), and Timulak and Keogh (2016) enhance the accessibility, interest value, and application potential of our analysis of the case of Cora. We hope that readers will take the time to reflect on these commentaries, and we applaud the journal for making it possible for us to present our work in a dialogical, collegial setting. We strongly believe that the creation of practical knowledge is a collective endeavor, and that the meaning of any single piece of research only comes to life when the points of connections that extend between it, and other sources of ideas and evidence, are made visible. In an ideal or future world, we would hope that all readers would be able to take part in this kind of dialogical process.

\section{REFERENCES}

Altman, A. J. \& Miller, R. B. (2016). Considering “Cora”: A critical appreciation. Pragmatic Case Studies in Psychotherapy, 12(3), Article 4, 215-223. Available:

pcsp.libraries.rutgers.edu.

Angus, L., Goldman, R., \& Mergenthaler, E. (2008). Introduction. One case, multiple measures: An intensive case-analytic approach to understanding client change processes in evidence-based, emotion-focused therapy of depression. Psychotherapy Research, 18, 629-633. doi: org/10.1080/10503300802430673

Ben-Shar, A. R. \& Shalit, R. (eds) (2016). When hurt remains. Relational perspectives on therapeutic failure. London: Karnac.

Benelli, E., De Carlo, A., Biffi, D., \& McLeod, J. (2015). Hermeneutic Single Case Efficacy Design: A systematic review. TPM - Testing, Psychometrics, Methodology in Applied Psychology, 22, 97-133. doi: 10.4473/TPM22.1.7

Bohart, A. C. (2016). The life-saving case of "Cora": A Rogerian perspective. Pragmatic Case Studies in Psychotherapy, 12(3), Article 2, 194-206. Available: pcsp.libraries.rutgers.edu 
Bramley, N. \& Eatough, V. (2005). The experience of living with Parkinson's disease: An interpretative phenomenological analysis case study. Psychology \& Health, 20, 223- 235. doi: 10.1080/08870440412331296053

Craig, P., Dieppe, P., Macintyre, S., Michie, S., Nazareth, I., \& Petticrew, M. (2008). Developing and evaluating complex interventions: the new Medical Research Council guidance. British Medical Journal, 337:a1655. doi: 10.1136/bmj.a1655

Eatough, V. \& Smith, J. A. (2006a). I feel like a scrambled egg in my head: An idiographic case study of meaning making and anger using interpretative phenomenological analysis. Psychology and Psychotherapy: Theory, Research and Practice, 79, 115-135. doi: 10.1348/147608305X41100

Eatough, V., \& Smith, J. A. (2006b). I was like a wild wild person: Understanding feelings of anger using interpretative phenomenological analysis. British Journal of Psychology, 97, 483-498. doi: 10.1348/000712606X97831

Elliott, R. E. (2002). Hermeneutic Single Case Efficacy Design. Psychotherapy Research, 12, 1-20. doi: $10.1080 / 713869614$

Fishman, D.B., \& Westerman, M.A. (2011). A key role for case studies: Theory building. Pragmatic Case Studies in Psychotherapy, 7(4), Article 1, 434-439. Available: pcsp.libraries.rutgers.edu doi: http://dx.doi.org/10.14713/pcsp.v7i4.1111

Flyvbjerg, B. (2006). Five misunderstandings about case-study research. Qualitative Inquiry, 12, 219-245. doi: 10.1177/1077800405284363

Geller, J. \& Farber, B. (1993). Factors influencing the process of internalization in psychotherapy. Psychotherapy Research, 3, 166-180. doi: 10.1080/10503309312331333769

Gergen, K. (1997). Who speaks and who replies in human science scholarship? History of the Human Sciences, 10, 151-173. doi: 10.1177/095269519701000311

Greenberg, L. S. (2008). The case of Lisa: An integration and reaction to the process analyses, Psychotherapy Research, 18, 677-682. doi: 10.1080/10503300802441530

Halvorsen, M.S., Benum, K., Haavind, H., \& McLeod, J. (2016). A life-saving therapy: The theory-building case of "Cora." Pragmatic Case Studies in Psychotherapy, 12(3), Article 1, 158-193. Available: pcsp.libraries.rutgers.edu.

Hill, C. E., Sim, W., Spangler, P., Stahl, J., Sullivan, C., \& Teyber, E. (2008). Therapist immediacy in brief psychotherapy: Case study II. Psychotherapy, 45, 3, 298-315. doi: 10.1037/a0013306

Knox, S., Goldberg, J. L., Woodhouse, S. S., \& Hill, C. E. (1999). Clients’ internal representations of their therapists. Journal of Counseling Psychology, 46, 244-256. doi: 10.1037/0022-0167.46.2.244

Liotti, G. (2006). A model of dissociation based on attachment theory and research. Journal of trauma \& Dissociation, 4, 55-74. doi: 10.1300/J229v07n04_04

Lyons-Ruth, K., Dutra, L., Schuder, M. R., \& Bianchi, I. (2006). From infant attachment disorganization to adult dissociation. Psychiatric clinics of North America, 29 (1), 63-86. doi: 10.1016/j.psc.2005.10.011

McLeod, J. (2010). Case study research in counselling and psychotherapy. London: Sage. McLeod, J. (2011). Qualitative research in counseling and psychotherapy (2 ${ }^{\text {nd }}$ ed.). London: Sage. 
McLeod. J. (2013). Increasing the rigor of case study evidence in therapy research. Pragmatic Case Studies in Psychotherapy, 9(4), Article 1, pp. 382-402. Available: pcsp.libraries.rutgers.edu. doi: http://dx.doi.org/10.14713/pcsp.v9i4.1832

Mosher, J. K. \& Stiles, W. B. (2009). Clients' assimilation of experiences of their therapists. Psychotherapy, 46, 432-447. doi: 10.1037/a0017955

Ogden, P. \& Fisher, J. (2015). Sensorimotor psychotherapy. Interventions for trauma and attachment. New York: W. W. Norton \& Company.

Plsek, P.E. \& Greenhalgh, T. (2001). Complexity science. The challenge of complexity in health care. British Medical Journal, 323, 625-628.

Porges, S. W. (2001). The polyvagal theory. Neuro physiological foundations of emotions, attachment, communication and self-regulation. New York: W. W. Norton \& Company.

Råbu, M., Haavind, H., \& Binder, P.-E. (2013). We have travelled a long distance and sorted out the mess in the drawers: Metaphors for moving towards the end in psychotherapy. Counselling and Psychotherapy Research, 13, 71- 80. doi: 10.1080/14733145.2012.711339

Quinn, M.C., Schofield, M.J., \& Middleton, W. (2012). Successful psychotherapy for psychogenic seizures in men. Psychotherapy Research, 22, 682-698. doi: 10.1080/10503307.2012.704085

Shine, L., \& Westacott, M. (2010). Reformulation in cognitive analytic therapy: Effects on the working alliance and the client's perspective on change. Psychology and Psychotherapy: Theory, Research and Practice, 83, 161-177. doi:10.1348/147608309X471334

Shore, A. N. (2003). Affect regulation and the repair of the self. New York: W. W. Norton \& Company.Slife, B.D. \& Gantt, E.E. (1999). Methodological pluralism: a framework for psychotherapy research. Journal of Clinical Psychology, 55, 1453-1465. doi: 10.1002/(SICI)1097-4679(199912)55:12<1453::AID-JCLP4>3.0.CO;2-C

Solbakken, O. A., Hansen, R. S., \& Monsen, J. T. (2011). Affect integration and reflective function: Clarification of central conceptual issues. Psychotherapy Research, 21 (4), 482496. doi: 10.1080/10503307.2011.583696

Stangeland, T. (2012). Når sikreste behandling ikke er beste behandling. [When safest treatment is not best treatment]. Tidsskrift for Norsk Psykologforening, 49, 138-144.

Stiles, W. B. (2007). Theory-building case studies of counselling and psychotherapy. Counselling and Psychotherapy Research, 7(2), 122-127. doi:10.1080/14733140701356742

Swedberg, R. (2014). The Art of Social Theory. New Jersey, NJ: Princeton University Press.

Timulak, L. \& Keogh, D. (2016). The Case of "Cora": Clinical and methodological perspectives. Pragmatic Case Studies in Psychotherapy, 12(3), 207-214. Available: pcsp.libraries.rutgers.edu

Wall, J.M., Kwee, J.L., Hu, M., \& McDonald, M.J. (2016). Enhancing the hermeneutic singlecase efficacy design: Bridging the research-practice gap. Psychotherapy Research. Published online. doi: 10.1080/10503307.2015.1136441

Wilson, T., \& Holt, T. (2001). Complexity science. Complexity and clinical care. British Medical Journal, 323, 685-688. 